\title{
Intragraft donor-specific anti-HLA antibodies in phenotypes of chronic lung allograft dysfunction
}

\author{
Annelore Sacreas ${ }^{1}$, Jean-Luc Taupin ${ }^{2}$, Marie-Paule Emonds ${ }^{3,4}$, \\ Liesbeth Daniëls $^{3}$, Dirk E. Van Raemdonck ${ }^{1,5}$, Robin Vos (id) ${ }^{1}$, Geert M. Verleden ${ }^{1}$, \\ Bart M. Vanaudenaerde ${ }^{1}$, Antoine Roux ${ }^{6}$, Stijn E. Verleden ${ }^{1}$ and the Leuven \\ Lung Transplant Group ${ }^{1,7}$
}

Affiliations: ' ${ }^{1}$ Leuven Lung Transplant Group, Dept of Chronic Diseases, Metabolism and Ageing (CHROMETA), KU Leuven, Leuven, Belgium. ${ }^{2}$ Laboratoire d'Immunologie et Histocompatibilité, Saint-Louis Hospital, Paris, France. ${ }^{3}$ Histocompatibility and Immunogenetics Laboratory, Belgian Red Cross-Flanders, Mechelen, Belgium. ${ }^{4}$ Dept of Immunology and Microbiology, KU Leuven, Leuven, Belgium. ${ }^{5}$ Dept of Thoracic Surgery, University Hospitals Leuven, Leuven, Belgium. 'S Service de Transplantation Pulmonaire, Foch Hospital, Suresnes, France. ${ }^{7} \mathrm{~A}$ list of members of the Leuven Lung Transplant Group can be found in the Acknowledgements section.

Correspondence: Stijn E. Verleden, Laboratory of Respiratory Diseases, Dept of Chronic Diseases, Metabolism and Ageing (CHROMETA), KU Leuven, Herestraat 49, Box 706, 3000 Leuven, Belgium.

E-mail: stijn.verledenđakuleuven.be

@ERSpublications

Discrepancies between serological and pathological/clinical findings in DSAs after lung transplantation are common. This article provides evidence that graft DSA assessment yields complementary information for lung transplant patients. http://bit.ly/2GIkae1

Cite this article as: Sacreas A, Taupin J-L, Emonds M-P, et al. Intragraft donor-specific anti-HLA antibodies in phenotypes of chronic lung allograft dysfunction. Eur Respir J 2019; 54: 1900847 [https://doi. org/10.1183/13993003.00847-2019].

\section{ABSTRACT}

Introduction: Circulating anti-human leukocyte antigen (HLA) serum donor-specific antibodies (sDSAs) increase the risk of chronic lung allograft dysfunction (CLAD) and mortality. Discrepancies between serological and pathological/clinical findings are common. Therefore, we aimed to assess the presence of tissue-bound graft DSAs (gDSAs) in CLAD explant tissue compared with sDSAs.

Methods: Tissue cores, obtained from explant lungs of unused donors $(n=10)$ and patients with bronchiolitis obliterans syndrome (BOS; $n=18$ ) and restrictive allograft syndrome (RAS; $n=18$ ), were scanned with micro-computed tomography before elution of antibodies. Total IgG levels were measured via ELISA. Anti-HLA class I and II IgG gDSAs were identified using Luminex single antigen beads and compared with DSAs found in serum samples.

Results: Overall, mean fluorescence intensity was higher in RAS eluates compared with BOS and controls $(\mathrm{p}<0.0001)$. In BOS, two patients were $\mathrm{sDSA}^{+} / \mathrm{gDSA}^{+}$and two patients were $\mathrm{sDSA}^{-} / \mathrm{gDSA}^{+}$. In RAS, four patients were $\mathrm{sDSA}^{+} / \mathrm{gDSA}^{+}$, one patient was $\mathrm{sDSA}^{+} / \mathrm{gDSA}^{-}$and five patients were $\mathrm{sDSA}^{-} / \mathrm{gDSA}^{+}$. Serum and graft results combined, DSAs were more prevalent in RAS compared with BOS (56\% versus 22\%; $\mathrm{p}=0.04$ ). There was spatial variability in gDSA detection in one BOS patient and three RAS patients, who were all sDSA ${ }^{-}$. Total graft IgG levels were higher in RAS than BOS $(\mathrm{p}<0.0001)$ and in $\mathrm{gDSA}^{+}$versus $\mathrm{gDSA}^{-}(\mathrm{p}=0.0008)$, but not in $\mathrm{sDSA}^{+}$versus $\mathrm{sDSA}^{-}(\mathrm{p}=0.33)$. In RAS, total IgG levels correlated with fibrosis $(\mathrm{r}=-0.39 ; \mathrm{p}=0.02)$.

Conclusions: This study underlines the potential of gDSA assessment as complementary information to sDSA findings. The relevance and applications of gDSAs need further investigation. 


\section{Introduction}

Survival after allograft transplantation, irrespective of allograft type, remains problematic. This is partly due to the complexity of combining two genomes (that of the donor and that of the recipient) and the ability of the recipient's immune system to recognise the allograft as nonself, causing a proliferative alloimmune response that leads to allograft rejection and dysfunction. Of all solid organ transplantations, lung allografts fare the worst, with chronic lung allograft dysfunction (CLAD) affecting 50\% of primary lung transplant recipients within 3-5 years following transplantation $[1,2]$.

CLAD is defined as a persistent decline $\geqslant 20 \%$ in forced expiratory volume in $1 \mathrm{~s}\left(\mathrm{FEV}_{1}\right)$ [3] and chronic rejection is suspected when no identifiable cause for CLAD is found. Further phenotyping is made based on pulmonary function and imaging [4], being either obstructive, i.e. bronchiolitis obliterans syndrome (BOS), or restrictive accompanied by persistent computed tomography (CT) opacities, i.e. restrictive allograft syndrome (RAS) [5, 6]. Moreover, patients with RAS experience a worse prognosis (1-1.5 years) compared with patients with BOS (3-5 years) [7-9].

Recognition of donor human leukocyte antigens (HLAs) as nonself and the subsequent production of anti-HLA donor-specific antibodies (DSAs) is a predominant driver of alloimmune response after transplantation $[10,11]$. This may lead to antibody-mediated rejection (AMR), resulting in graft injury through various DSA-driven mechanisms [12]. Circulating de novo DSAs are therefore a key diagnostic criterion for AMR after lung transplantation, next to characteristic lung histology with or without evidence of (endothelial) complement C4d deposition within the graft [13].

Occurring in $10-50 \%$ of lung transplant recipients, post-transplant de novo DSAs increase the risk of CLAD and mortality [14-16]. Moreover, an important difference in prognosis between persistent and transient DSAs was demonstrated, as well as an association between DSA/AMR and the RAS phenotype $[17,18]$. Unfortunately, detection and interpretation of circulating DSAs (serum DSAs (sDSAs)) remains challenging, and discrepancies between serological and pathological/clinical findings are common. It has already been suggested that sDSAs may not always be detectable during AMR in kidney and liver transplantation [19-21]. In lung transplant biopsies, Visentin et al. [22] demonstrated that sDSAs in the presence of graft DSAs (gDSAs) reliably identified the most pathogenic DSAs and lung transplant recipients with a higher risk for graft loss, although without discerning BOS and RAS phenotype.

Therefore, in the present study, we assessed the presence and relevance of tissue-bound intragraft DSAs in well-characterised explant lungs of BOS and RAS patients.

\section{Materials and methods \\ Study cohort}

36 redo lung transplantations and autopsies performed in CLAD patients at University Hospitals Leuven (Leuven, Belgium) from October 2010 to November 2017 were included. RAS was diagnosed based on a restrictive pulmonary function (either forced vital capacity (FVC) decline $\geqslant 20 \%$ or total lung capacity (TLC) decline $\geqslant 10 \%$ compared with the best baseline post-lung transplantation) in combination with the presence of persistent opacities on chest CT [4]. In all other cases, BOS was diagnosed. Clinical phenotype was confirmed via histopathological analysis of the contralateral lung (i.e. normal parenchyma and obliterative bronchiolitis in BOS or interstitial fibrosis and pleural/septal thickening with/without obliterative bronchiolitis in RAS). 10 declined human donor lungs were used as controls (reason for decline: persistent embolisation $n=4$, infection $n=2$, contusion $n=2$, kidney tumour $n=1$ and unexpected death of recipient $\mathrm{n}=1$ ).

\section{Ethics statement}

Written informed consent to participate in biobanking and scientific research was provided by all patients included in the study. This was approved by the Institutional Review Board of University Hospitals Leuven and under existing Belgian law stating that organs of insufficient quality for transplantation can be used in approved research programmes (S57742, S51577 and ML6385).

\section{Explant lungs}

Human explant lungs were collected at the time of redo lung transplantation or autopsy, as described previously [23]. Air-inflated lungs were frozen in liquid nitrogen fumes, and cores of $1.4 \mathrm{~cm}$ diameter and $2 \mathrm{~cm}$ length were extracted. For this study, 18 BOS and 18 RAS lungs were used from which two cores from distinctly separate regions (different locations within the lung and for RAS at different degrees of fibrosis as assessed by micro-CT) were taken to investigate regional disparity within the lung; one tissue core was used in the control group. All tissue cores were scanned in the frozen condition $\left(-30^{\circ} \mathrm{C}\right)$ using micro-CT (SkyScan 1172; Bruker, Kontich, Belgium). Surface density was assessed by CTAN (Bruker) and used as a read-out of disease severity, as lower values reflect increased tissue fibrosis [24]. 
Elution and measurement of IgG antibodies from biopsies

Tissue (the size of a needle biopsy; $\sim 112 \mathrm{~mm}^{3}$ ) was extracted from each tissue core and homogenised [22]. Intragraft IgG antibodies were eluted using a Gamma ELU-KIT II (Immucor, Norcross, GA, USA). Total IgG levels were measured via sandwich ELISA (Invitrogen, Waltham, MA, USA), according to the manufacturer's instructions.

Anti-HLA antibody testing via Luminex

Anti-HLA class I and II antibodies were identified in graft eluates using single antigen HLA tests (LABScreen LS1A04 and LS2A01; One Lambda, Canoga Park, CA, USA) on a Luminex 200 analyser (Luminex, Austin, TX, USA), according to the manufacturer's instructions. The mean fluorescence intensity (MFI) values were normalised to the MFI of the negative control beads. Donor specificity was determined via identification of the specificity of HLA antibodies and donor HLA typing. The positivity threshold for gDSAs was set at MFI $>75$ and higher than the mean +5 SD of the MFI obtained in graft eluates for the recipient's own alleles, calculated for each HLA locus. This method has been refined since the original article by VisEnTIN et al. [22]. The positivity threshold is lower for eluates than for serum, due to a lower nonspecific background caused by the eluate matrix when compared with raw serum.

\section{sDSA assessment}

sDSAs profiles were assessed for all patients [17]. In University Hospitals Leuven, routine measurements are performed at lung transplantation, at month 1, 3, 6, 9, 12, 18 and 24 post-transplant, and yearly thereafter. Additional measurements are performed based on clinical information. Luminex was used according to the institution's protocol to detect anti-HLA antibodies. The positivity threshold for sDSA was set at MFI 500. When a screening was considered positive, identification of the specificity of HLA antibodies using single antigen beads followed to define it as a DSA or not (Immucor). A distinction was made between de novo persistent sDSAs, i.e. present against the same donor HLA antigen on at least two measurements at $\geqslant 3$ weeks apart, and cleared sDSAs, i.e. present against the donor HLA antigen for $<3$ weeks with all subsequent measurements being negative.

\section{Statistical analysis}

Results are presented as mean with standard error of the mean or median (interquartile range (IQR)). Discrete data were compared via contingency tables and Chi-squared tests. For continuous data, the Mann-Whitney U-test or two-way ANOVA with Šídák's multiple comparisons was used. The log-rank test was used for survival analysis and correlation was performed using Spearman's rank test. Presence of sDSAs and gDSAs was compared per patient. As two cores per lung were assessed, patient gDSA positivity was defined by at least one core with gDSAs. For IgG and surface density, results were analysed per core. Prism version 8.0 (GraphPad, San Diego, CA, USA) was used. $\mathrm{p}<0.05$ was considered significant.

\section{Results}

\section{Study cohort characteristics}

Study cohort characteristics are shown in table 1. There were no demographic differences between control, BOS and RAS subjects except for donor age ( $p=0.04$, not attributable to specific group). Median graft survival post-CLAD diagnosis was worse in RAS compared with BOS ( $\mathrm{p}=0.0073$ ). Last $\mathrm{FEV}_{1}$ and $\mathrm{FEV}_{1} /$ FVC were lower in BOS compared with RAS ( $\mathrm{p}=0.0047$ and $\mathrm{p}<0.0001$, respectively), while FVC and TLC were lower in RAS compared with BOS ( $\mathrm{p}=0.0053$ and $\mathrm{p}=0.013$, respectively).

\section{Control lung tissue}

Among the 10 control samples, one sample showed increased median (IQR) MFI for all HLA loci compared with the other samples (65.5 (25.25-84.00) versus 11 (8-14); $\mathrm{p}<0.0001)$ (data not shown) without any explanation. However, none of the MFI values reached the positivity threshold for antibodies.

\section{General MFI strength in graft eluates}

Overall, the normalised mean MFI was higher in RAS eluates compared with BOS and controls $(\mathrm{p}<0.0001)$, and was locus dependent $(\mathrm{p}<0.0001)$. Specifically for HLA-C and HLA-DQ, median (IQR) MFI was significantly higher in RAS (42 (11-263) and 66 (4-332), respectively) than in BOS (22 (9-102) and $22(12-82)$, respectively; $\mathrm{p}<0.0001)$ and controls $(16(7-26)$ and $16(8-22)$, respectively; $\mathrm{p}<0.0001)$ (figure $1 \mathrm{a})$.

\section{DSAs in serum and graft}

A total of 11 patients (31\%) (three BOS patients (27\%) and eight RAS patients (73\%)) had positive measurements for sDSAs. Seven (64\%; two BOS and five RAS) of these patients had persistent sDSAs, while three (27\%; one BOS and two RAS) had cleared sDSAs and one (9\%; RAS) only had sDSAs in the last measurement prior to redo lung transplantation. Patients who cleared the sDSAs after a brief period of 
TABLE 1 Study cohort characteristics

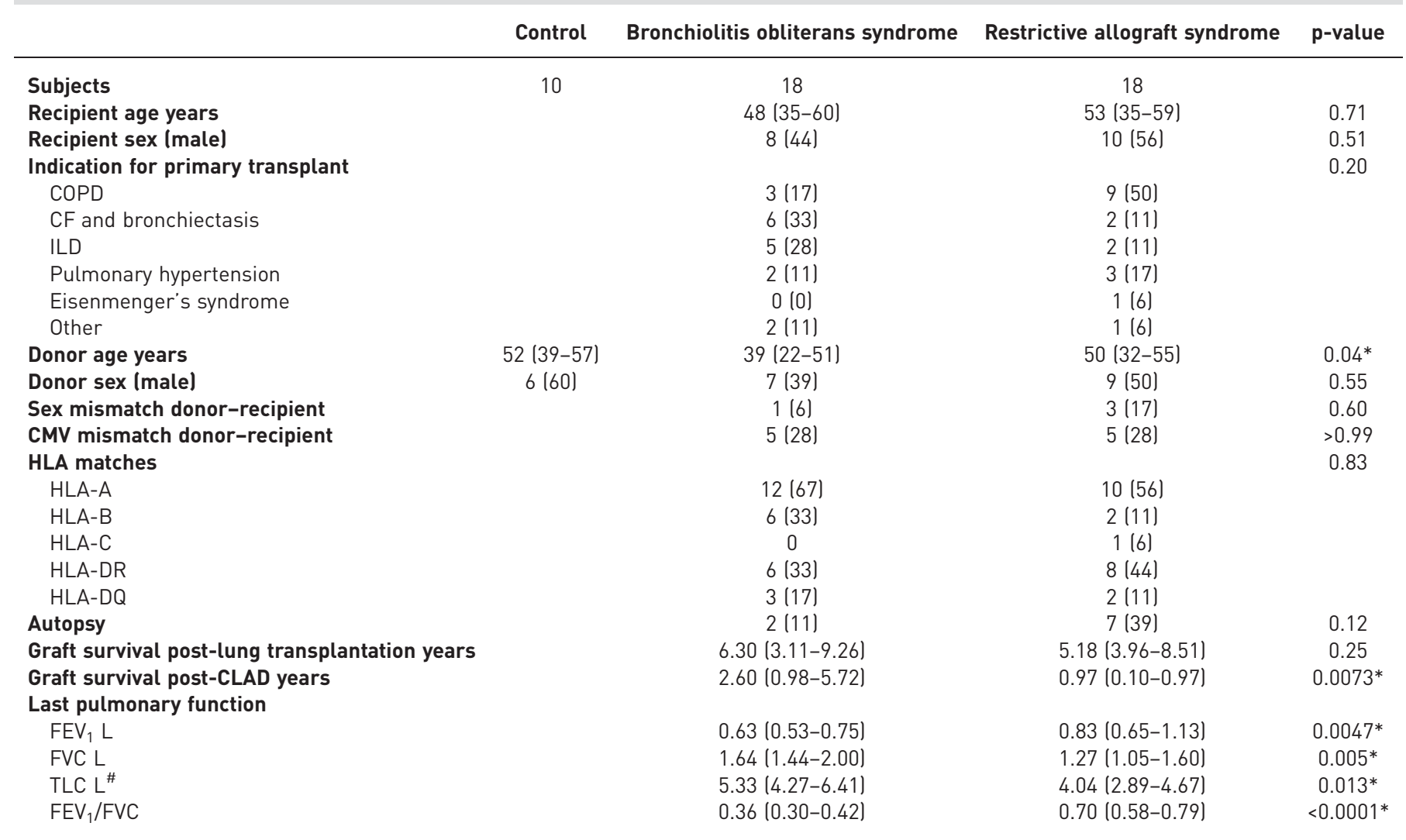

Data are presented as $\mathrm{n}$, median (interquartile range) or $\mathrm{n}(\%)$, unless otherwise stated. COPD: chronic obstructive pulmonary disease; CF: cystic fibrosis; ILD: interstitial lung disease; CMV: cytomegalovirus; HLA: human leukocyte antigen; CLAD: chronic lung allograft dysfunction; $\mathrm{FEV}_{1}$ : forced expiratory volume in $1 \mathrm{~s}$; FVC: forced vital capacity; TLC: total lung capacity. \#: TLC data were missing for six BOS patients and seven RAS patients. p-values show the results of the Kruskal-Wallis ANOVA or Mann-Whitney test in the case of continuous data. For discrete data, the results of the contingency table are shown. *: $p<0.05$ was considered significant.
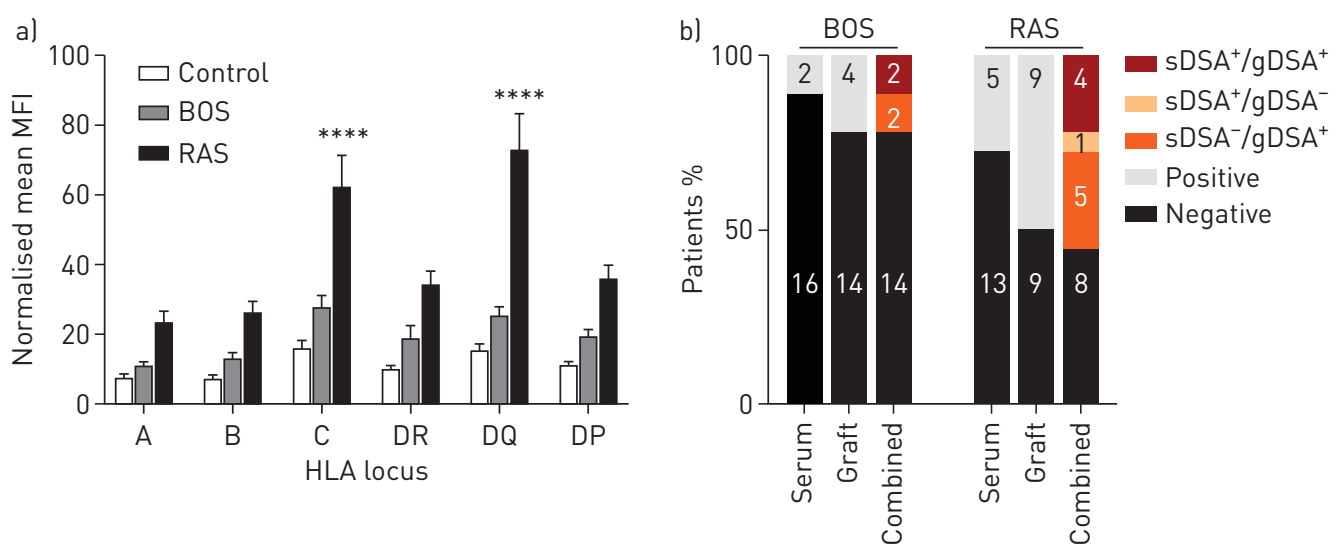

FIGURE 1 Mean fluorescence intensity (MFI) values of anti-human leukocyte antigen (HLA) class I and II antibodies and prevalence of donor-specific antibodies (DSAs) in chronic lung allograft dysfunction tissue. BOS: bronchiolitis obliterans syndrome; RAS: restrictive allograft syndrome; sDSA: serum DSA; gDSA: graft DSA. a) Overview of MFI values of control ( $n=10), B O S(n=18)$ and RAS ( $n=18)$ graft eluates, presented as mean \pm SEM, showing increased and locus-dependent MFI levels in RAS compared with BOS and controls. Specifically for HLA-C and HLA-DQ, MFI was significantly higher in RAS than BOS and controls. ****: $p<0.0001$. b) Prevalence of DSA in serum, in the graft or combined, for BOS and RAS patients, presented as percentage of total patients with absolute numbers indicated on the bar graph. 
positivity or only had the last measurement positive prior to redo lung transplantation were considered as $\mathrm{sDSA}^{-}$for further analyses.

In BOS, the two patients with persistent sDSAs also exhibited gDSAs ( $\mathrm{sDSA}^{+} / \mathrm{gDSA}^{+}(11 \%)$ ) (figure $1 \mathrm{~b}$ ). Two other BOS patients were negative for sDSAs but positive for gDSAs (sDSA ${ }^{-} / \mathrm{gDSA}^{+}(11 \%)$ ). The patient with cleared sDSAs did not show any gDSAs. Among the four gDSA ${ }^{+}$patients, one (25\%) displayed anti-HLA class I targeting HLA-A, one (25\%) displayed anti-HLA class I and II targeting HLA-B and HLA-DR, and two had anti-HLA class II targeting HLA-DQ (n=2 (50\%)) (table 2).

In RAS, five patients had persistent sDSAs after transplantation. Of these patients, four also showed gDSAs $\left(\mathrm{sDSA}^{+} / \mathrm{gDSA}^{+}(22 \%)\right.$ ), while one was negative for $\mathrm{gDSAs}\left(\mathrm{sDSA}^{+} / \mathrm{gDSA}^{-}(6 \%)\right.$ ) (figure $\left.1 \mathrm{~b}\right)$. Five RAS patients were negative for persistent sDSAs but positive for gDSAs ( $\mathrm{sDSA}^{-} / \mathrm{gDSA}^{+}(28 \%)$ ), of which one had cleared sDSAs. The majority of gDSAs found in RAS patients were against HLA class II: four targeting HLA-DQ (44\%) and one targeting both HLA-DR and HLA-DQ (11\%) (table 2). Two were against HLA class I targeting HLA-B $(n=1(11 \%))$ and HLA-C $(n=1(11 \%))$, whereas two patients had gDSAs against both class I and class II targeting HLA-A $(\mathrm{n}=1(11 \%))$ or HLA-C $(\mathrm{n}=1(11 \%))$ as well as HLA-DQ.

Therefore, independent of CLAD phenotype, seven $\mathrm{sDSA}^{-}$patients demonstrated gDSA positivity and one $\mathrm{SDSA}^{+}$patient was gDSA ${ }^{-}$. Serum and graft results combined, DSAs were significantly higher in RAS compared with BOS ( $56 \%$ versus $22 \%$; $\mathrm{p}=0.04$ ) (figure $1 \mathrm{~b}$ ). However, follow-up time (6.30 versus 5.18 years; $\mathrm{p}=0.25$ ) (table 1 ) and time to first sDSA detection (5.97 versus 3.40 years; $\mathrm{p}=0.62$ ) were similar between the groups.

\section{Spatial variability of DSA presence in situ}

Given the discrepancy between sDSAs and gDSAs for many patients, we investigated the possibility that gDSAs could be focal, resulting in one core being positive for gDSAs while the other is negative within the same lung. In BOS, one out of the four $\mathrm{gDSA}^{+}$patients $(25 \%)$ showed intragraft variability, whereas in RAS this was observed for three out of the nine gDSA $^{+}$patients $(33 \%)$ (figure $2 \mathrm{a}$ ). Interestingly, all of these patients were $\mathrm{sDSA}^{-}$. This was not associated with morphological findings as evident from micro-CT (increased fibrosis, obliterative bronchiolitis lesions, etc.) (figure $2 \mathrm{~b}-\mathrm{e}$ ).

\section{Total IgG levels and correlation with morphology}

To further investigate this spatial variability, total IgG levels were determined in graft eluates as a measure of immunological activity. Total graft IgG was significantly higher in RAS versus BOS (median (IQR) 660

TABLE 2 Human leukocyte antigen (HLA) antigenic specificities of donor-specific antibodies (DSAs) in serum

\begin{tabular}{|c|c|c|c|c|c|c|c|c|c|c|c|c|c|c|}
\hline \multirow{2}{*}{$\begin{array}{c}\text { sDSA/ } \\
\text { gDSA } \\
\text { status }\end{array}$} & \multirow{2}{*}{$\begin{array}{c}\text { C4d } \\
\text { status } \\
\text { in TBB }\end{array}$} & \multirow{2}{*}{$\begin{array}{c}\text { First } \\
\text { sDSA }^{+} \\
\text {POY }\end{array}$} & \multirow{2}{*}{$\begin{array}{c}\text { Last } \\
\text { sDSA+ } \\
\text { POY }\end{array}$} & \multicolumn{5}{|c|}{ Serum } & \multicolumn{5}{|c|}{ Graft } & \multirow{2}{*}{$\begin{array}{c}\text { Explant } \\
\text { or } \\
\text { autopsy } \\
\text { POY }\end{array}$} \\
\hline & & & & A & B & C & DR & $D Q$ & A & B & C & DR & $D Q$ & \\
\hline
\end{tabular}

\begin{tabular}{|c|c|c|c|c|c|c|c|c|c|c|c|c|c|c|c|}
\hline \multicolumn{16}{|l|}{ BOS } \\
\hline 1 & $+/+$ & NA & 15.83 & 16.99 & 0 & 0 & 0 & DR15 & 0 & 0 & 0 & 0 & 0 & DQ6/8; DQA1*05 & 17.18 \\
\hline 2 & $-/+$ & NA & NA & NA & 0 & 0 & 0 & 0 & 0 & 0 & B51 & 0 & DR15/51 & 0 & $7.18^{\#}$ \\
\hline 3 & $+/+$ & NA & 2 & 6.88 & 0 & 0 & 0 & DR52 & DQ7 & A25 & 0 & 0 & 0 & 0 & 6.92 \\
\hline 5 & $-1-$ & - & NA & NA & 0 & 0 & 0 & 0 & 0 & 0 & 0 & 0 & 0 & 0 & 1.68 \\
\hline \multicolumn{16}{|l|}{ RAS } \\
\hline 1 & $-/+$ & NA & NA & NA & 0 & 0 & 0 & 0 & 0 & 0 & 0 & 0 & 0 & $D Q 5 / 6$ & 8.71 \\
\hline 4 & $+/-$ & NA & 1.53 & 4.06 & 0 & 0 & 0 & DR15 & DQ2 & 0 & 0 & 0 & 0 & 0 & 4.30 \\
\hline 5 & $+/+$ & NA & 6.05 & 8.45 & 0 & 0 & 0 & DR52 & DQ2 & 0 & 0 & 0 & 0 & DQ2 & 8.45 \\
\hline 6 & $-/+$ & NA & 8.01 & 8.01 & 0 & 0 & 0 & 0 & 0 & 0 & 0 & 0 & DR8/52 & DQ7/8/9 & $9.07^{\#}$ \\
\hline 7 & $-1+$ & NA & NA & NA & 0 & 0 & 0 & 0 & 0 & 0 & B35 & 0 & 0 & 0 & $8.16^{\#}$ \\
\hline 8 & $-/+$ & - & 3.56 & 3.56 & 0 & 0 & 0 & 0 & 0 & A3 & 0 & 0 & 0 & DQ7/8/9 & $4.13^{\#}$ \\
\hline 9 & $+/+$ & - & 0.73 & 1.5 & 0 & 0 & 0 & DR51 & DQ5/6 & 0 & 0 & Cw7 & 0 & DQ5/6 & $1.84^{\#}$ \\
\hline
\end{tabular}

sDSA: serum; gDSA: graft DSA; C4d: complement component 4d; TBB: transbronchial biopsy; POY: post-operative year; BOS: bronchiolitis obliterans syndrome; NA: not applicable; RAS: restrictive allograft syndrome. ${ }^{\#}$ : autopsy. 

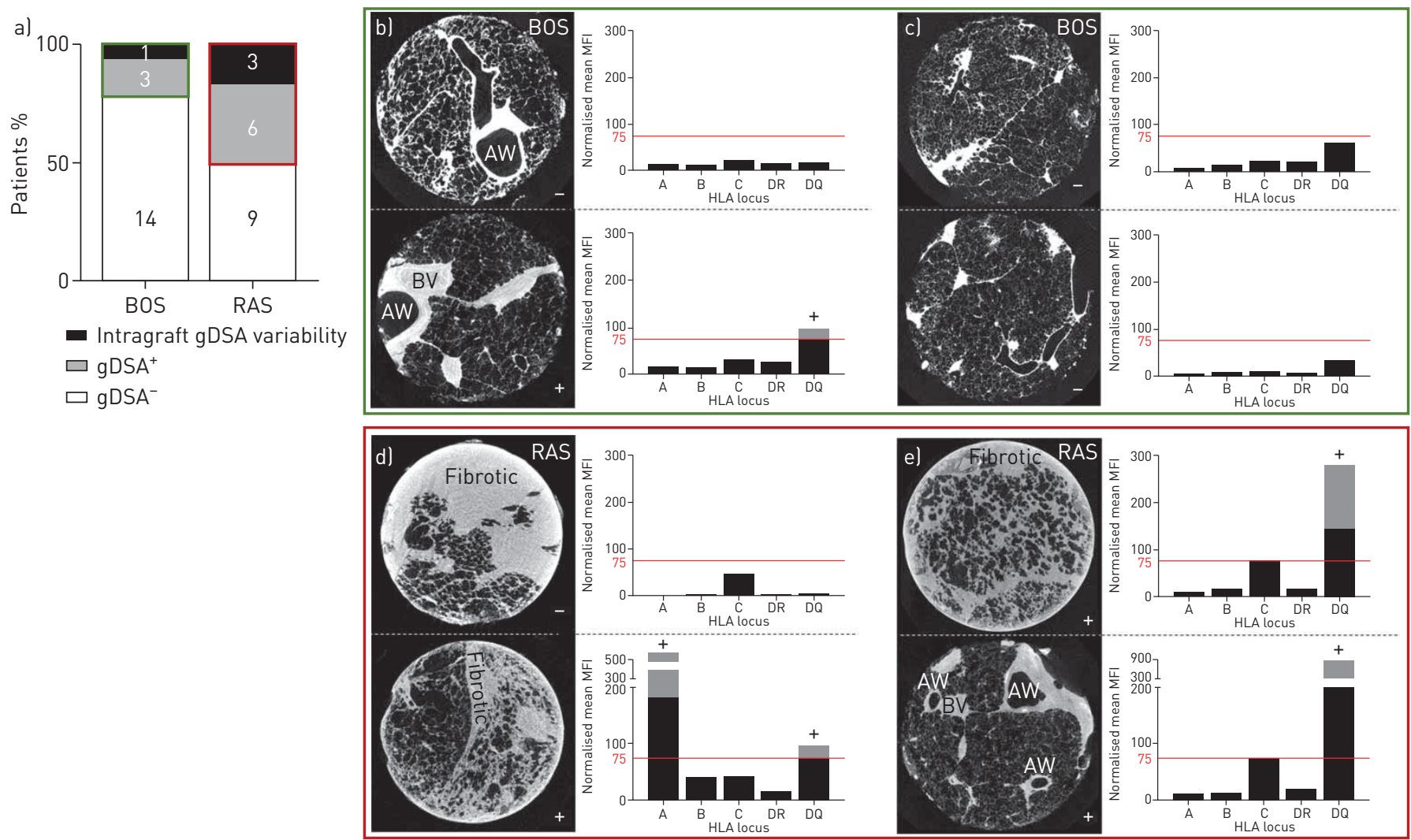

FIGURE 2 Spatial graft donor-specific antibody (DSA) variability within lungs. RAS: restrictive allograft syndrome; gDSA: graft DSA; BOS: bronchiolitis obliterans syndrome; CT: computed tomography; MFI: mean fluorescence intensity; AW: airway; BV: blood vessel. Per lung, two cores from distinctly separate regions (apical versus basal) were taken to investigate regional disparity within the lung. Within RAS, different degrees of fibrosis were also taken into account. a) Spatial variability of gDSA positivity was detected within the same lung for one BOS patient and three RAS patients. b, c) Micro-CT images of two cores from two BOS lungs b) with and c) without spatial gDSA variability. d, e) Micro-CT images of two cores from RAS lungs d) with and e) without spatial gDSA variability. Each micro-CT image is accompanied by the MFI values of the donor alleles; the red line shows the limit of MFI $>75$, while the grey shading represents MFI greater than mean+5SD. gDSA positivity in a HLA locus is indicated by a "+" sign above the bar.

(489-914) versus 220 (190-463) ng. $\mathrm{mL}^{-1}$; p <0.0001) (figure 3a). Independent of the CLAD phenotype, total IgG concentration was higher in $\mathrm{gDSA}^{+}$samples versus $\mathrm{gDSA}^{-}$samples (median (IQR) 624 (4541017) versus $\left.292(173-654) \mathrm{ng} \cdot \mathrm{mL}^{-1} ; \mathrm{p}=0.0008\right)$ (figure $3 \mathrm{~b}$ ). There was no difference in total $\operatorname{IgG}$ concentration in $\mathrm{sDSA}^{+}$and $\mathrm{sDSA}^{-}$samples (median (IQR) 541 (396-679) versus $336(213-774) \mathrm{ng} \cdot \mathrm{mL}^{-1}$; $\mathrm{p}=0.33$ ) (figure 3c).

As there was a pronounced difference in the degree of fibrosis in RAS samples, we further used micro-CT to quantify surface density, used as a measure of degree of fibrosis. Total IgG levels and surface density showed a weak inverse correlation in RAS $(r=-0.39 ; \mathrm{p}=0.02)$ (figure $3 \mathrm{~d}$ ). However, when discriminating $\mathrm{gDSA}^{+}$and $\mathrm{gDSA}^{-}$RAS samples, the significance was lost $\left(\mathrm{gDSA}^{+}\right.$samples: $\mathrm{r}=-0.5 ; \mathrm{p}=0.06 ; \mathrm{gDSA}^{-}$ samples: $r=-0.26 ; \mathrm{p}=0.26$ ) (figure $3 \mathrm{e}$ and $\mathrm{f}$ ).

C4d staining was performed for two (11\%) BOS and six (33\%) RAS patients. In BOS, both patients were C4d negative ( $\mathrm{sDSA}^{-} / \mathrm{gDSA}^{-}, \mathrm{n}=1 ; \mathrm{sDSA}^{-} / \mathrm{gDSA}^{+}, \mathrm{n}=1$ ). In RAS, two patients were $\mathrm{C} 4 \mathrm{~d}$ positive $\left(\mathrm{sDSA}^{-} /\right.$ $\left.\mathrm{gDSA}^{-}, \mathrm{n}=1 ; \mathrm{sDSA}^{+} / \mathrm{gDSA}^{+}, \mathrm{n}=1\right)$ and four were C4d negative $\left(\mathrm{sDSA}^{-} / \mathrm{gDSA}^{-}, \mathrm{n}=2 ; \mathrm{sDSA}^{-} / \mathrm{gDSA}^{+}, \mathrm{n}=1\right.$; $\left.\mathrm{sDSA}^{+} / \mathrm{gDSA}^{+}, \mathrm{n}=1\right)$.

Due to clinical suspicion of AMR, three (17\%) BOS and six (33\%) RAS patients had previously received treatment consisting of a 3-day course of high-dose intravenous steroids, rituximab (one dose of $\left.375 \mathrm{mg} \cdot \mathrm{m}^{-2}\right)$ and $7-10$ sessions of plasmapheresis, followed by i.v. immunoglobulins $\left(0.5 \mathrm{mg} \cdot \mathrm{kg}^{-1}\right)$ after each session. In BOS, two patients were $\mathrm{sDSA}^{-} / \mathrm{gDSA}^{-}$, whereas one was $\mathrm{sDSA}^{-} / \mathrm{gDSA}^{+}$. In RAS, three patients were $\mathrm{sDSA}^{-} / \mathrm{gDSA}^{-}$, one was $\mathrm{sDSA}^{-} / \mathrm{gDSA}^{+}$and two were $\mathrm{sDSA}^{+} / \mathrm{gDSA}^{+}$.

Case report demonstrates DSA detection in the graft prior to appearance in serum

To further demonstrate the importance of gDSA assessment, an illustrative case is shown in figure 4 . At 8 years post-lung transplant, the patient developed RAS, without detectable sDSAs, for which he 

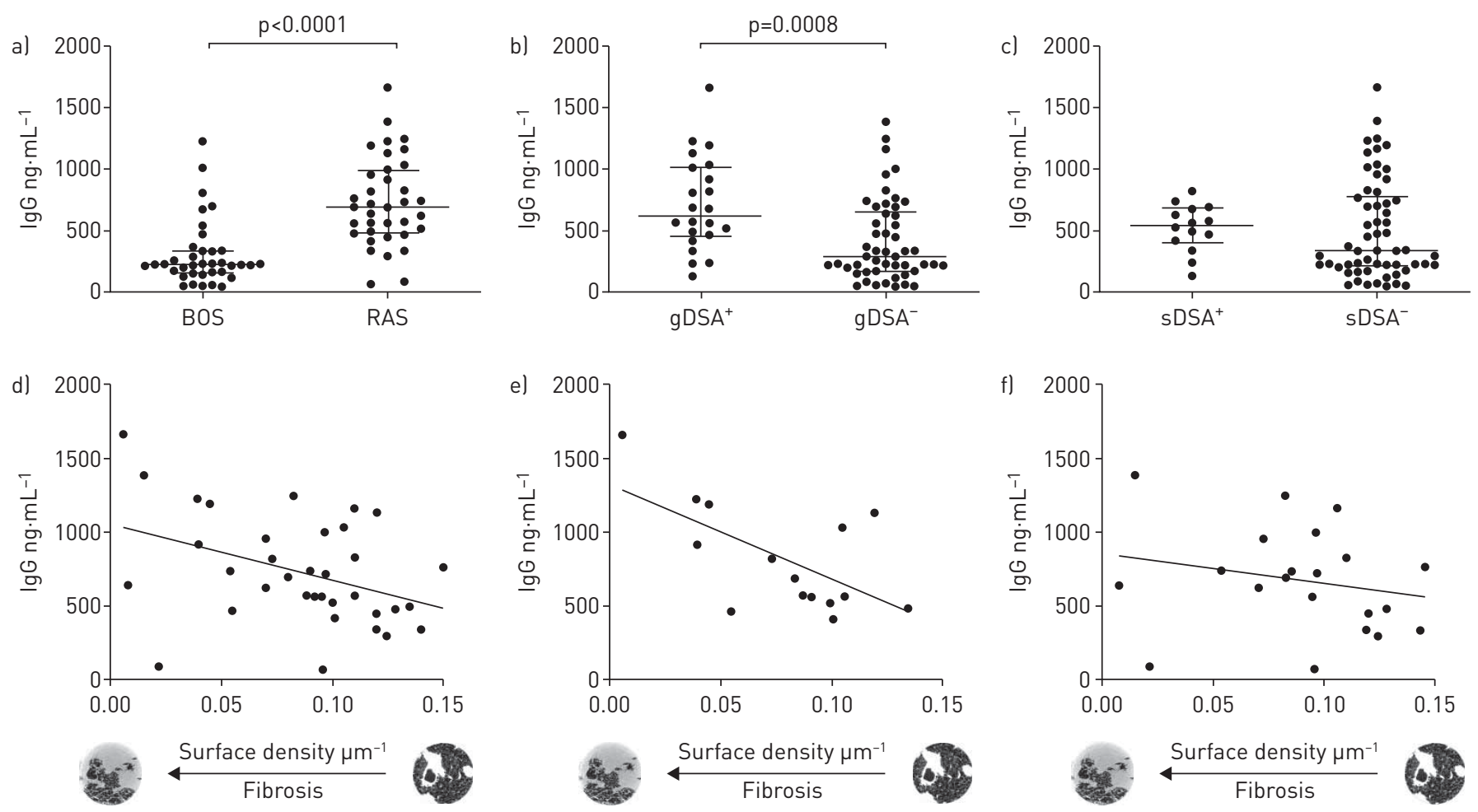

FIGURE 3 Total IgG levels and correlation with morphology. BOS: bronchiolitis obliterans syndrome; RAS: restrictive allograft syndrome; gDSA: graft DSA: sDSA: serum DSA. Total IgG levels, presented with median and interquartile range, in a) BOS ( $n=36$ ) versus RAS ( $=36$ ) cores showing increased IgG levels in RAS ( $p<0.0001)$, b) gDSA $(n=22)$ versus $g_{D S A}{ }^{-}(n=50)$ cores, independent of CLAD phenotype, showing increased IgG levels in $\mathrm{gDSA}^{+}$cores ( $\left.\mathrm{p}=0.0008\right)$, and $\left.c\right) \mathrm{sDSA}^{+}(\mathrm{n}=14)$ versus $\mathrm{sDSA}^{-}(\mathrm{n}=58)$ cores, showing no significant difference between groups. Surface density was used as a measure of the amount of fibrosis, with lower values reflecting a higher degree of fibrosis. d) In RAS samples, surface density as assessed by CTAN inversely correlated with total lgG levels $(r=-0.39 ; p=0.02)$; there was a trend towards significance in $e) g D S A^{+}(r=-0.5 ; p=0.06)$ but not in $f) g D S A^{-}(r=-0.26 ; p=0.26)$ RAS samples.

underwent redo lung transplantation. The patient re-developed CLAD and de novo DSAs targeting HLA-DQ1 of the first donor were detected in serum 6 years following redo lung transplantation. Our retrospective analysis of the explanted first lung, obtained at redo lung transplantation, demonstrated that the anti-HLA-DQ1 DSAs against the first donor were already detectable in the graft at the time of redo lung transplantation, without ever detecting circulating DSAs earlier.

\section{Discussion}

In this study, we confirm that DSAs can be detected in graft eluates from lung allograft tissue, supporting previous findings [22]. We report that DSAs, whether in serum or in the graft, were more prominent in RAS than BOS in our cohort. Interestingly, sDSA negativity did not necessarily mean gDSA negativity or vice versa: our findings show seven $\mathrm{sDSA}^{-} / \mathrm{gDSA}^{+}$patients and one $\mathrm{sDSA}^{+} / \mathrm{gDSA}^{-}$patient. Moreover, gDSA positivity was subject to spatial variability within the lungs, especially in patients without sDSAs. Total IgG levels were higher in RAS and in $\mathrm{gDSA}^{+}$samples, and inversely correlated with surface density in RAS.

The overall higher MFI levels in RAS tissue suggest a higher humoral baseline compared with BOS. Graft total IgG levels were also higher in RAS, which is consistent with earlier studies showing increased complement proteins and immunoglobulins (pointing to activation of B-cells) in bronchoalveolar lavage of RAS patients, whereas lymphoid follicles were only present in RAS tissue [25, 26]. Taken together, these results reinforce the idea that the humoral immune system is predominantly important in RAS.

Our results indicate a clinical relevance of gDSA assessment complementary to sDSAs, especially in RAS. The high prevalence of DSA positivity in RAS supports previous statements of possible RAS and AMR overlap [17, 18], since some patients diagnosed with RAS might actually be concealed (chronic) AMR, which has implications for therapy such as plasmapheresis and i.v. immunoglobulin treatment. When attempting to relate the DSA results with C4d staining data, no associations could be found. However, although the most recent consensus on pulmonary AMR includes C4d positivity in the diagnostic criteria, the sensitivity of C4d staining is questioned [13]. Moreover, several studies have indicated that C4d 


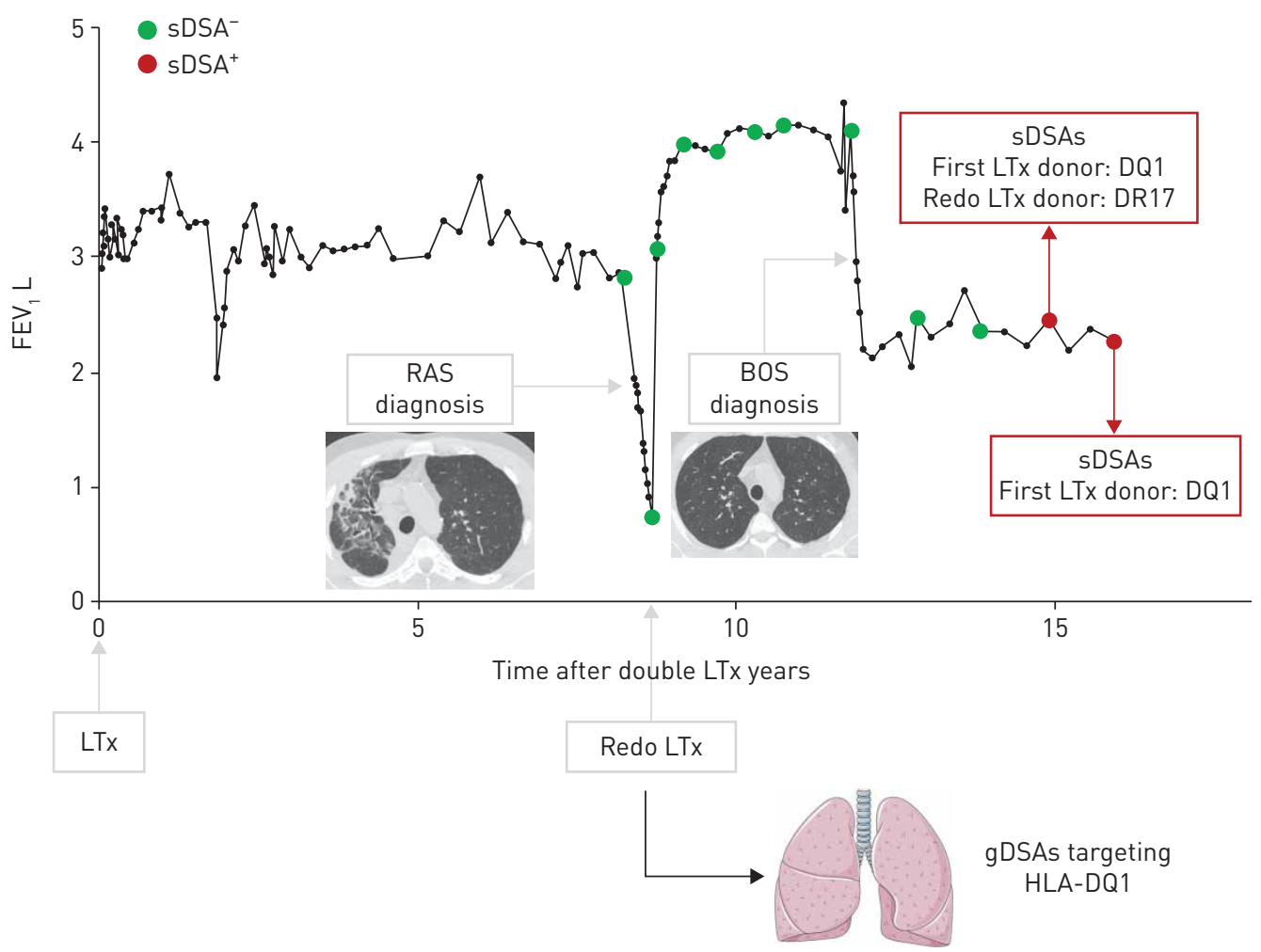

FIGURE 4 Case report demonstrating donor-specific antibody (DSA) detection in the graft prior to appearance in serum. $\mathrm{FEV}_{1}$ : forced expiratory volume in $1 \mathrm{~s} ; \mathrm{LTx}$ : lung transplantation; RAS: restrictive allograft syndrome; BOS: bronchiolitis obliterans syndrome; SDSA: serum DSA; HLA: human leukocyte antigen. Evolution in $\mathrm{FEV}_{1}$ of a lung transplant patient. At 8 years post-LTx, the patient developed RAS, without sDSAs being detected. After redo LTx, the patient re-developed chronic lung allograft dysfunction type BOS. De novo serum DSAs were not detected in serum until 6 years post-redo LTx (anti-HLA-DQ1). However, the DSAs targeting HLA-DQ1 were already detectable in the graft obtained at the time of redo LTx.

staining is difficult to interpret in lung biopsies, due to poor reproducibility, high background staining and insufficient sensitivity for AMR [27-30].

We were able to detect regional disparity of gDSA positivity within the same lung for one BOS patient and three RAS patients. This might not be surprising if we keep the heterogeneity of disease severity within CLAD lungs, and specifically within RAS, in mind. Besides, a single biopsy cannot be representative of a whole organ, as is the case with a tissue core and lung. Another possible explanation for this heterogeneity could be the in situ antibody production in bronchus-associated lymphoid tissue. Interestingly, no sDSAs were detected in any of these patients, which may be due to strong intragraft binding (i.e. the sponge effect of the capillary bed), reducing sDSA levels.

Measuring the total IgG levels and demonstrating that these were not only higher in RAS versus BOS, but also in $\mathrm{gDSA}^{+}$versus $\mathrm{gDSA}^{-}$samples, underlines the immunological importance of this spatial variability and renders support to our finding of significant heterogeneity. Total IgG levels in RAS also inversely correlated with surface density, implying that eluates obtained from more fibrotic tissue held more IgG antibodies than nonfibrotic or less fibrotic tissue.

Given that our findings demonstrated seven $\mathrm{sDSA}^{-} / \mathrm{gDSA}^{+}$patients, this suggests that in situ DSA assessment from the graft could increase the sensitivity of DSA detection in lung transplantation patients. This could be crucial when thinking of therapies, but also when considering cross-matching before redo lung transplantation. Our case example, where we report detection of gDSAs against HLA-DQ1 prior to detection in serum, further supports this notion.

There are some limitations to this study. First, one could scrutinise our positivity definition in grafts, suggesting that we are overestimating the number of gDSAs. Indeed, defining the optimal positivity threshold for grafts proved challenging as there is limited literature to start from. We chose to use the MFI values obtained in graft eluates for the alleles expressed by the recipient (i.e. the recipient's self-antigens) 
as a baseline per locus. The positivity threshold was then set at MFI $>75$ and higher than mean $+5 \mathrm{SD}$ of the recipients self-antigens, a method adapted from the studies of BACHELET et al. [31] and VisENTIN et al. [22]. Furthermore, the stringency of the positivity threshold was tested in a subanalysis, where MFI $>75$ (as previously mentioned) but a cut-off of mean+3SD of the recipients self-antigens was used. Including these possible gDSAs resulted in one extra $\mathrm{sDSA}^{-} / \mathrm{gDSA}^{+} \mathrm{BOS}$ patient, one extra $\mathrm{sDSA}^{-} / \mathrm{gDSA}^{+} \mathrm{RAS}$ patient and one extra $\mathrm{sDSA}^{+} / \mathrm{gDSA}^{+}$RAS patient. All these patients showed spatial gDSA variability within the lung. However, this subanalysis showed significantly more false positives (i.e. non-DSA anti-HLA antibodies) and, therefore, the definition using mean+5SD was adopted for further analyses.

Secondly, samples were obtained from explants at redo lung transplantation as well as from autopsies, meaning there could be a delay in the processing of some samples even though our centre aims to process all autopsies within $24 \mathrm{~h}$. Nonetheless, MFI values did not differ between explant or autopsy and gDSAs were demonstrated in both groups.

Another criticism could be that sDSAs were not properly removed during the washing procedure before elution and that what we are measuring as gDSAs are in fact remnants of sDSAs, i.e. merely a serum dilution. However, this method has been used previously and can be considered validated [22]. Moreover, the supernatant of the last washing step can be used as a negative control to demonstrate that all serum antibodies have been removed, which has been verified in previous work [22]. Also, some patients showed completely different DSAs in serum than in the graft, whereas others showed only one of the sDSAs in their graft tissue. We believe that there are two possible scenarios leading to these differences: 1) in situ antibody production in bronchus-associated lymphoid tissue, resulting in completely distinct DSAs in serum and the graft, and 2) a difference in binding capacity between the sDSA within the lung allograft, resulting in some but not all of the sDSA found in the graft.

Although gDSA assessment seems promising, there are several issues to contemplate before its routine use in the clinic. In addition to the time required to elute and analyse gDSAs, the interpretation remains challenging and unstandardised. We should also note that the size of tissue used in this study might be larger than what would be possible in the clinical setting. Furthermore, the regional disparity in gDSA positivity shows that it might be necessary to take several biopsies from distinct regions. Transbronchial biopsies may therefore not be ideal to work with, given their small size and limited range.

In conclusion, this study underlines the potential of gDSA assessment as complementary information to sDSA findings. However, further investigation is needed about whether stable lung transplant recipients demonstrate gDSAs to determine the relevance and clinical applications of gDSAs.

Acknowledgements: The authors wish to thank and acknowledge P. De Leyn, P. Nafteux, H. Decaluwé, H. Van Veer, L.J. Ceulemans and L. Depypere (Dept of Thoracic Surgery, University Hospital Gasthuisberg, Leuven, Belgium) for providing explanted lung tissue.

The Leuven Lung Transplant Group includes: Anke Van Herck, Janne Kaes, Tobias Heigl, Sofie Ordies, Laurens J. De Sadeleer, Arno Vanstapel, Arne P. Neyrinck, Veronique Schaevers, Lieven J. Dupont, Jonas Yserbyt, Laurent Godinas, Lieven Depypere, Anna E. Frick, Laurens J. Ceulemans, Eric K. Verbeken, Birgit Weynand, Paul De Leyn, Willy Coosemans, Hans Van Veer, Philippe Nafteux and Herbert Decaluwé.

Support statement: R. Vos is senior clinical research fellow of the Research Foundation Flanders (FWO), Belgium $(12 \mathrm{G} 8715 \mathrm{~N})$ and is supported by a research grant of UZ Leuven, Belgium (STG15/023). S.E. Verleden is a post-doctoral research fellow of the Research Foundation Flanders (FWO 12G8715N and 1500617N), Belgium and is supported by a research grant from the International Society for Heart and Lung Transplantation and the KU Leuven (C24/18/073)

Conflict of interest: A. Sacreas has nothing to disclose. J-L. Taupin has nothing to disclose. M-P. Emonds has nothing to disclose. L. Daniëls has nothing to disclose. D.E. Van Raemdonck has nothing to disclose. R. Vos has nothing to disclose. G.M. Verleden has nothing to disclose. B.M. Vanaudenaerde has nothing to disclose. A. Roux reports personal fees and nonfinancial support from Biotest, personal fees from Thermo Fisher, nonfinancial support from CSL Berhing, outside the submitted work. S.E. Verleden has nothing to disclose.

\section{References}

1 Kulkarni HS, Cherikh WS, Chambers DC, et al. Bronchiolitis obliterans syndrome-free survival after lung transplantation: an International Society for Heart and Lung Transplantation Thoracic Transplant Registry analysis. J Heart Lung Transplant 2019; 38: 5-16.

2 Chambers DC, Cherikh WS, Goldfarb SB, et al. The International Thoracic Organ Transplant Registry of the International Society for Heart and Lung Transplantation: thirty-fifth adult lung and heart-lung transplant report - 2018; focus theme: multiorgan transplantation. J Heart Lung Transplant 2018; 37: 1169-1183.

3 Verleden GM, Glanville AR, Lease ED, et al. Chronic lung allograft dysfunction: definition, diagnostic criteria, and approaches to treatment - a consensus report from the Pulmonary Council of the ISHLT. J Heart Lung Transplant 2019; 38: 493-503. 
4 Glanville AR, Verleden GM, Todd JL, et al. Chronic lung allograft dysfunction: definition and update of restrictive allograft syndrome - a consensus report from the Pulmonary Council of the ISHLT. J Heart Lung Transplant 2019; 38: 483-492.

5 Ofek E, Sato M, Saito T, et al. Restrictive allograft syndrome post lung transplantation is characterized by pleuroparenchymal fibroelastosis. Mod Pathol 2013; 26: 350-356.

6 Verleden GM, Raghu G, Meyer KC, et al. A new classification system for chronic lung allograft dysfunction. J Heart Lung Transplant 2014; 33: 127-133.

7 Verleden SE, Ruttens D, Vandermeulen E, et al. Bronchiolitis obliterans syndrome and restrictive allograft syndrome: do risk factors differ? Transplantation 2013; 95: 1167-1172.

8 Sato M, Waddell TK, Wagnetz U, et al. Restrictive allograft syndrome (RAS): a novel form of chronic lung allograft dysfunction. J Heart Lung Transplant 2011; 30: 735-742.

9 Todd JL, Jain R, Pavlisko EN, et al. Impact of forced vital capacity loss on survival after the onset of chronic lung allograft dysfunction. Am I Respir Crit Care Med 2014; 189: 159-166.

10 Valenzuela NM, Reed EF. Antibody-mediated rejection across solid organ transplants: manifestations, mechanisms, and therapies. J Clin Invest 2017; 127: 2492-2504.

11 Loupy A, Lefaucheur C. Antibody-mediated rejection of solid-organ allografts. $N$ Engl J Med 2018; 379: $1150-1160$.

12 McCaughan JA, Tinckam KJ. Donor specific HLA antibodies \& allograft injury: mechanisms, methods of detection, manifestations and management. Transpl Int 2018; 31: 1059-1070.

13 Levine DJ, Glanville AR, Aboyoun C, et al. Antibody-mediated rejection of the lung: a consensus report of the International Society for Heart and Lung Transplantation. J Heart Lung Transplant 2016; 35: 397-406.

14 Snyder LD, Wang Z, Chen DF, et al. Implications for human leukocyte antigen antibodies after lung transplantation: a 10-year experience in 441 patients. Chest 2013; 144: 226-233.

15 Safavi S, Robinson DR, Soresi S, et al. De novo donor HLA-specific antibodies predict development of bronchiolitis obliterans syndrome after lung transplantation. J Heart Lung Transplant 2014; 33: 1273-1281.

16 Tikkanen JM, Singer LG, Kim SJ, et al. De novo DQ donor-specific antibodies are associated with chronic lung allograft dysfunction after lung transplantation. Am J Respir Crit Care Med 2016; 194: 596-606.

17 Verleden SE, Vanaudenaerde BM, Emonds MP, et al. Donor-specific and -nonspecific HLA antibodies and outcome post lung transplantation. Eur Respir J 2017; 50: 1701248.

18 Roux A, Bendib Le Lan I, Holifanjaniaina S, et al. Antibody-mediated rejection in lung transplantation: clinical outcomes and donor-specific antibody characteristics. Am J Transplant 2016; 16: 1216-1228.

19 Del Bello A, Congy N, Sallusto F, et al. Anti-human leukocyte antigen immunization after early allograft nephrectomy. Transplantation 2012; 93: 936-941.

20 Del Bello A, Congy-Jolivet N, Sallusto F, et al. Donor-specific antibodies after ceasing immunosuppressive therapy, with or without an allograft nephrectomy. Clin J Am Soc Nephrol 2012; 7: 1310-1319.

21 Neau-Cransac M, Le Bail B, Guidicelli G, et al. Evolution of serum and intra-graft donor-specific anti-HLA antibodies in a patient with two consecutive liver transplantations. Transpl Immunol 2015; 33: 58-62.

22 Visentin J, Chartier A, Massara L, et al. Lung intragraft donor-specific antibodies as a risk factor for graft loss. J Heart Lung Transplant 2016; 35: 1418-1426.

23 Verleden SE, Vasilescu DM, Willems S, et al. The site and nature of airway obstruction after lung transplantation. Am J Respir Crit Care Med 2014; 189: 292-300.

24 Verleden SE, McDonough JE, Verleden GM, et al. Phenotypical diversity of airway morphology in chronic lung graft vs. host disease after stem cell transplantation. Mod Pathol 2019; 32: 817-829.

25 Vandermeulen E, Schols D, Emonds M-P, et al. Humoral immunity in phenotypes of chronic lung allograft dysfunction: a broncho-alveolar lavage fluid analysis. Transpl Immunol 2016; 38: 27-32.

26 Vandermeulen E, Lammertyn E, Verleden SE, et al. Immunological diversity in phenotypes of chronic lung allograft dysfunction: a comprehensive immunohistochemical analysis. Transpl Int 2017; 30: 134-143.

27 Aguilar PR, Carpenter D, Ritter J, et al. The role of C4d deposition in the diagnosis of antibody-mediated rejection after lung transplantation. Am J Transplant 2018; 18: 936-944.

28 Roden AC, Maleszewski JJ, Yi ES, et al. Reproducibility of complement 4d deposition by immunofluorescence and immunohistochemistry in lung allograft biopsies. J Heart Lung Transplant 2014; 33: 1223-1232.

29 Roden AC, Aisner DL, Allen TC, et al. Diagnosis of acute cellular rejection and antibody-mediated rejection on lung transplant biopsies: a perspective from members of the pulmonary pathology society. Arch Pathol Lab Med 2017; 141: 437-444.

30 Westall GP, Snell GI, McLean C, et al. C3d and C4d deposition early after lung transplantation. J Heart Lung Transplant 2008; 27: 722-728.

31 Bachelet T, Couzi L, Lepreux S, et al. Kidney intragraft donor-specific antibodies as determinant of antibody-mediated lesions and poor graft outcome. Am J Transplant 2013; 13: 2855-2864. 